\title{
The "Time" to Timely Predict Ischemic Deficit After Subarachnoid Hemorrhage
}

\author{
Alfredo Conti - Francesco Tomasello
}

Published online: 7 February 2012

(C) Springer Science+Business Media, LLC 2012

Cerebral vasospasm still represents the leading cause of morbidity and mortality after subarachnoid hemorrhage (SAH). Nevertheless, ischemic deficits due to cerebral vasospasm are-at least in part-preventable. We know that vasospasm affects primarily patients with a major rupture resulting in an initial poor clinical condition, but it remains difficult to accurately and timely predict who, among patients with $\mathrm{SAH}$, will develop symptomatic vasospasm and eventually a cerebral infact or infarcts.

The only way to avoid possible disastrous delays in recognizing a decrease of cerebral perfusion is the continuous analysis of state of cerebral hemodynamics. We have some instruments to do this, some with detailed probing into brain physiology, but one simple tool-the transcranial Doppler Ultrasound (TCD) - developed about 3 decades ago [1], still remains the only system to monitor cerebral vasospasm: The expert operator knows that precious information on the status of cerebral hemodynamics is hidden in the indistinct noise produced by the TCD during vasospasm. Sometime, this is when the sound of TCD looses its unpleasant noisy character and becomes "musical", as a consequence of regular vibration of a spastic segment of an artery [1].

Spectral analysis of ultrasound echoes provides different quantitative measures, the most obvious of which is the velocity of blood flow. The quantification of the flow velocity (FV) can detect vasospasm, but this is insufficient for predicting its course and clinical relevance. In fact, an increase of the flow velocities over $120 \mathrm{~cm} / \mathrm{s}$ can be recorded in more than $70 \%$ of patients with aneurysmal

\footnotetext{
A. Conti $(\bowtie) \cdot$ F. Tomasello

Department of Neurosurgery, University of Messina,

Via Consolare Valeria 1, 98125 Messina, Italy

e-mail: alfredo.conti@unime.it
}

SAH, while the incidence of "clinical" vasospasm averages $30 \%$ half of whom will develop a stroke [2].

Therefore many TCD findings have been used in an attempt to predict outcome after SAH [3]. For example, an average rise in $\mathrm{FV}$ of more than $20 \mathrm{~cm} / \mathrm{s} /$ day between day 3-7 after SAH, a rapid early rise of FV (more than 25\%/ day), a mean maximal absolute rise in $\mathrm{FV}$ in the middle or anterior cerebral arteries of $65 \pm 5 \mathrm{~cm} / \mathrm{s}$ over a 24-h period, and a higher Lindegaard ratio $(6 \pm 0.3)$ have been noted in patients who develop a delayed ischemic deficit. Increased pulsatility index and poor cerebrovascular reactivity to acetazolamide have also been associated with incipient cerebral ischemia and poor prognosis. The loss of cerebrovascular reserve leads also to an impairment of cerebral autoregulation. Its determination-using the transient hyperemic response test-can distinguish patients who are at risk of developing neurological symptoms [4]. This latter observation was done by the group of the University of Cambridge, UK.

In this issue of Neurocritical Care, this leading group in the study of cerebral hemodynamics presents a novel hemodynamic index, the "time constant" of cerebral arterial bed. The time constant describes how fast the cerebral arterial bed, distal to the point of TCD insonation, is filled with blood after cardiac contraction. The authors continuously and simultaneously recorded FV and arterial blood pressure and used a relatively simple mathematical model to decode information hidden in the spectral display of the TCD. Indeed, the spectrum of the TCD reflects the flow velocities of each single blood cell moving into the brain arteries. The spectral distribution of different velocities is therefore expression of the arterial blood pressure during the different phases of the cardiac cycle, the section of the arteries of the cranial base, the resistance of the whole system to the downstreaming and blood outflow. 
The mathematical model, summarized in the appendix of this article, but extensively described in previous studies of this group [5, 6], efficiently describes the physiological interactions of the intracranial compartments and their volume and pressure changes during the cardiac cycle.

To measure the cerebral time constant, Authors obtained an evaluation of the arterial compliance and cerebrovascular resistance from the simultaneous sampling of the FV envelope and arterial blood pressure. Both arterial compliance and cerebrovascular resistance, however, are dependent on the cross-sectional area of the insonated vessel, a parameter that varies along with progression of vasospasm. Nevertheless, the interplay between the cerebral arterial compliance and the cerebrovascular resistance is independent from the radius of the vessel and can be used in longitudinal evaluation of vasospasm progression. This interplay between the arterial compliance and cerebrovascular resistance can be measured in seconds and was named "time constant".

Results of this preliminary study suggest that cerebral vasospasm causes significant shortening of this constant; also, these changes precede the increase in mean blood FV, thus potentially extending the window for a therapeutic intervention. The shortening of the cerebral time window is the result of a jointly reduction of arterial compliance of the affected cerebral artery during cerebral vasospasm, due to the increase of tension of arterial smooth muscles, and of cerebrovascular resistance due to dilatation of the arterioles distal to spastic section in response to the diminished local perfusion pressure. Thus, this new cerebrovascular index combines data on vasospasm with an evaluation of the status of cerebrovascular reserve. A simple measurement of FV would give only an idea of presence of vasospasm, whereas indices of autoregulation, such as the mean index (Mx) [7] reflect the status of cerebrovascular reserve.

There are limitations in this study, some of which are recognized by authors. One issue that is worth mentioning is that the linearity between arterial blood pressure and FV is not so obvious and that the system may include many nonlinearities not considered by the model adopted in this study [8]. The relationship between arterial blood pressure, cerebral blood flow, FV, and arterial diameter becomes increasingly complex with worsening cerebral vasospasm [3]. Nonetheless, there is clear evidence that the indices proposed by the group of Cambridge accurately describe cerebral hemodynamics and its modifications in many different clinical conditions.

\section{References}

1. Aaslid R, Nornes H. Musical murmurs in human cerebral arteries after subarachnoid hemorrhage. J Neurosurg. 1984;60:32-6.

2. Kassell NF, Haley EC Jr, Apperson-Hansen C, Alves WM. Randomized, double-blind, vehicle-controlled trial of tirilazad mesylate in patients with aneurysmal subarachnoid hemorrhage: a cooperative study in Europe, Australia, and New Zealand. J Neurosurg. 1996;84:221-8.

3. Aaslid R. Transcranial Doppler assessment of cerebral vasospasm. Eur J Ultrasound. 2002;16:3-10.

4. Lam JM, Smielewski P, Czosnyka M, Pickard JD, Kirkpatrick PJ. Predicting delayed ischemic deficits after aneurysmal subarachnoid hemorrhage using a transient hyperemic response test of cerebral autoregulation. Neurosurgery 2000;47:819-25; discussions 25-6.

5. Czosnyka M, Piechnik S, Richards HK, Kirkpatrick P, Smielewski P, Pickard JD. Contribution of mathematical modelling to the interpretation of bedside tests of cerebrovascular autoregulation. J Neurol Neurosurg Psychiatry. 1997;63:721-31.

6. Kim DJ, Kasprowicz M, Carrera E, et al. The monitoring of relative changes in compartmental compliances of brain. Physiol Meas. 2009;30:647-59.

7. Soehle M, Czosnyka M, Pickard JD, Kirkpatrick PJ. Continuous assessment of cerebral autoregulation in subarachnoid hemorrhage. Anesth Analg. 2004;98:1133-9.

8. Giller CA, Mueller M. Linearity and non-linearity in cerebral hemodynamics. Med Eng Phys. 2003;25:633-46. 